\title{
Interval-valued intuitionistic fuzzy sets as tools for evaluation of data mining processes
}

\author{
Krassimir Atanassov \\ Department of Bioinformatics and Mathematical Modelling \\ Institute of Biophysics and Biomedical Engineering, Bulgarian Academy of Sciences \\ 105 Acad. G. Bonchev Str., 1113 Sofia, Bulgaria, and \\ Intelligent Systems Laboratory, Prof. Asen Zlatarov University \\ Bourgas-8010, Bulgaria \\ e-mail: krat@bas.bg
}

Received: 15 September 2018

Accepted: 30 October 2018

\begin{abstract}
Intuitionistic Fuzzy Sets (IFSs), proposed in 1983, are extensions of fuzzy sets. Some years after their introduction, interval-valued IFSs (IVIFSs) were introduced. During the last 30 years, their properties were studied and these sets were used as tool for evaluation of different objects and processes from the area of the Artificial Intelligence. Short review of these legs of research is offered, with some concrete ideas of possible new directions of study. On this basis, a non-formal discussion is raised on the benefits of applying various elements of IVIFSs as tools for evaluation of Data Mining processes.
\end{abstract}

Keywords: Data mining, Interval-valued intuitionistic fuzzy set, Intuitionistic fuzzy set.

2010 Mathematics Subject Classification: $03 E 72$.

\section{Introduction}

This paper is a continuation of the author's paper [8]. Here, we discuss the origin, current state of research and applications in the area of Data Mining (DM) of one extension of Intuitionistic Fuzzy Sets (IFSs) and Logics (IFLs), called Interval-Valued IFSs (IVIFSs) and Logics (IVIFLs).

The first research, related to IVIFSs started in 1988 - 1989 [3,13]. Their basic definitions and the definitions of the operations, relations and operators, defined over them are described in [6] and in a series of papers, e.g., $[10,11]$. Here, we use some these definitions. 
The components in the IVIFS- and IVIFL-definitions give more and larger evaluating possibilities and determine the place of the IVIFSs and IVIFLs among the separate types of fuzzy sets. In the last 25 years the IVIFSs have been used for evaluating of processes in a wide range of areas, e.g. of Systems Theory (ST), Artificial Intelligence (AI) and Intelligent Systems (IS), medicine, chemical industry, ecology, etc.

Here we describe some of the IVIFS-applications in the AI and IS, and their benefits and discuss the possibilities for application of the IVIFSs as tools for evaluating of DM-processes.

\section{IVIFSs and Data Mining - possibilities for the future}

Following [8], we ask: "What is Data Mining"? The answer of this question is so unclear, as well as the answer of the question for the areas of the AI. Again, there are different answers in respect of the opinions of the specialists, giving answers. For example:

"The aim of DM is to make sense of large amounts of mostly unsupervised data, in some domain" [25];

"The aim of DM is to extract implicit, previously unknown and potentially useful (or actionable) patterns from data. DM consists of many up-to-date techniques such as classification (decision trees, naive Bayes classifier, $k$-nearest neighbor, NNs), clustering (k-means, hierarchical clustering, density-based clusteering), association (one-dimensional, multi-dimensional, multilevel association, constraint-based association)" [63];

"DM stands at the confluence of the fields of statistics and machine learning” [52];

"DM is a term that covers a broad range of techniques being used in a variety of industries" [50];

"DM is the core of the knowledge discovery in databases process, involving the inferring of algorithms that explore the data, develop the model and discover previously unknown patterns" [43].

$\mathrm{DM}$ is a process of finding reasonable correlations, repeating patterns and trends in large Data Bases (DBs) and Big Data (BD). As a basis of our research, we use the publications [18-21, 24-27, 29-31,33-35, 35-41, 43-53, 62, 63, 66, 67]. In the literature, different areas of the AI are determined as components of the DM. For example, the algorithms of decision making, pattern recognition, neural networks, genetic algorithms, etc.

Extending and modifying [5,8], here we make a review of some of the problems related to the above ones, those already existing, and those planned for future research. Everywhere we emphasize on:

- the way of the IVIFS-estimation of the process (object) up to now (if any);

- other ways for IVIFS-realization of this estimation;

- possible extensions or generalizations of already existing IFL-estimations of the corresponding processes (objects) and ways for their modifications. 


\subsection{IVIF-estimations in expert systems, data bases, data warehouses, big data, OLAP-structures}

As the author mentioned in [8], "A lot of colleagues already assert that the Expert Systems (ESs) are dying. The author supports the idea that they will live their "Renaissance", obtaining a special place in the instrumentation of DM. Preserving their basic purpose to generate a new knowledge by answering to hypotheses, we can essentially extend the area of their possibilities. When some unclear situation arises in a process controled by DM-tools, and when some hypotheses for its future development are generated, then the new type of ESs can help."

In [4], the concept of an Intuitionistic Fuzzy ES (IFES) was introduced. It was essentially extended in $[5,22,23,42]$. In these ESs, each fact $F$ has an IF-estimations $\langle\mu(F), \nu(F)\rangle$, determining its degrees of validity and non-validity. So, the answer whether a given hypothesis is valid or not, obtains essentially more precise evaluation. In near future, we will introduce an extension of the IFES which facts will have the IVIF-estimations $\langle M(F), N(F)\rangle$, where $M(F), N(F) \subseteq[0,1]$ and $\sup M(x)+\sup N(x) \leq 1$. So, we eilll define Interval-Valued IFES (IVIFES). A next step of the extensions will be the introduction of facts that contain moments of time, when they became valid, and moments in which they stopped being valid (a sequence of time-moments $t_{1}, t_{2}, \ldots, t_{n}$ ). Then (cf. [5]), on one hand, we can answer to time related questions ("at the moment", "once”, "sometimes", "for long/short time”, "often”, "rarely", "for short period", "for long period", etc.). On the other hand, the IVIFES rules can have essentially complex forms, containing different logical operations (conjunction, disjunction, implication, negation,...), quantifiers ("for existence" and "for all") and modal operators in their antecedents. In addition, the facts and rules can have priorities that will determine whether a given fact or rule can stay in the DB or must be changed with another one.

In future, the ES-answers can be additionally, so they can have optimistic, pessimistic, or another form. Similar directions for extensions of the DBs, Data Warehouses (DWs), BD, OLAPstructures, etc. can be realized.

As we assumed in [8], writing for IFESs and now - for IVIFESs, solving each of the above problems or, of course, all of them, will promote not only the theory and application of IVIFSs, but also the research in the area of DM, too.

In the next section, an example is given that can be used as an illustration for determining of $M$ - and $N$-evaluations of the facts.

\subsection{IVIF-estimations of a procedure for inductive reasoning}

As it is mentioned in [31], "the rule induction is one of the fundamental tools of DM. Usually rules are expressions of the form

$$
\begin{gathered}
\text { if }\left(\text { attribute }_{1}, \text { value }_{1}\right) \&\left(\text { attribute }_{2}, \text { value }_{2}\right) \& \ldots \&\left(\text { attribute }_{n}, \text { value }_{n}\right) \\
\text { then (decision, value)." }
\end{gathered}
$$

If we use the IFLs tools, we obtain sequentially

$$
\text { (attribute }_{1}, \text { value }_{1}, \mu_{1}, \nu_{1} \text { ), }
$$




$$
\begin{gathered}
\left(\text { attribute }_{2}, \text { value }_{2}, \mu_{2}, \nu_{2}\right), \\
\ldots \\
\text { (attribute } \left._{n}, \text { value }_{n}, \mu_{n}, \nu_{n}\right),
\end{gathered}
$$

where, in the simplest case

$$
\mu_{i}= \begin{cases}1, & \text { if } \text { value }_{i} \text { is anticipated (expected, correct, etc.) } \\ 0, & \text { if } \text { value }_{i} \text { is not anticipated (not expected, incorrect, etc.) } \\ *, & \text { if there is no information about attribute }\end{cases}
$$

and $\nu_{i}=1-\mu_{i}$, but in more general case $\mu_{i}, \nu_{i} \in[0,1]$ and $\mu_{i}+\nu_{i} \leq 1$.

Now, the final estimation have the IVIF-form (decision, value, $M, N$ ), where $M, N \subseteq[0,1]$ and $\sup M+\sup N \leq 1$.

Let $p$ be the number of degrees $\mu_{i}$ that are equal to $1, q$ be the number of degrees $\nu_{i}$ that are equal to $1, r$ be the number of degrees $\mu_{i}$ that satisfy $1>\mu_{i}>\frac{1}{2}, s$ be the number of degrees $\nu_{i}$ that satisfy $1>\nu_{i}>\frac{1}{2}$. Obviously, $p+q+r+s \leq n$.

Now, the final estimation can have the IVIF-form (decision, value, $M, N$ ), where

$$
M=\left[\frac{p}{n}, \frac{p+r}{n}\right], \quad N=\left[\frac{q}{n}, \frac{q+s}{n}\right] .
$$

Therefore, $M, N \subseteq[0,1]$ and $\sup M+\sup N \leq 1$.

Hence, we obtain more precise estimation for the validity of the procedure for inductive reasoning than the cases of standard, fuzzy and intuitionistic fuzzy inductive reasoning. If in the beginning we determine some threshold of validity $t_{v}$, then we can assert that a decision is positive sufficiently valid, if $\sup M>t_{v}$ and it is strongly positive sufficiently valid, if inf $M>t_{v}$. On the other hand, if we determine some treshold of non-validity $t_{n}$ then we can assert that a decision is negative sufficiently valid, if $\inf N<t_{n}$ and it is is strongly negative sufficiently valid, if $\sup N<t_{n}$.

\subsection{IVIF-estimations in decision making procedures}

The procedures for decision making include multi-criteria decision making procedures, that can be re-organized so that they to use IVIF-estimations. For example, let us have $s$ experts who must estimate some object or process. Let $m$ of them estimate it as "perfect", "the best" or "very good"; $n$ of them - as "worst" or "very bad"; $r$ - as "good", "suitable" or "useful"; and $s$ are "bad", "unsuitable" or "useless", then we can estimate the object or process by IVIF-estimations, using the formulas from the previous Section.

In [8], a new type of decision making procedure is discussed, based on the apparatus of the intercriteria analysis (see, e.g., $[12,14])$. It is called intercriteria decision making. Its aim is to find dependences among the used criteria. For example, it is very suitable when separate experts offer different criteria for use in concrete procedure. Now, after finishing of the procedure, we can determine whether there are connections between some of these criteria. In IFS-case, this procedure is discussed in [17], while for the IVIFS-case similar research is appeared. The new method is based on the apparatus of the index matrices (see $[2,7]$ ). 


\subsection{IVIFS-estimations in pattern recognition procedures}

The apparatus of the IVIFSs is suitable for estimation of different pattern recognition procedures. Here, we give the following two short examples, inspired by [9].

Example 1. Let us have the original pattern - in our example, triangle $A B C$ that must be compared to an other pattern - e.g., triangle $A F G$ (see Fig. 1). Let the section $B C$ be fuzzified, i.e., it be modified to the region $B C E D$.

Let us denote by $\# X$ the surface of region $X$ and let

$$
\begin{gathered}
s=\# A B H G, \\
a=\# A F I E, \\
b=\# F D I, \\
c=\# D B H I, \\
d=\# C H E, \\
e=\# C E I H .
\end{gathered}
$$

Obviously, $a+b+c+d+e=s$.

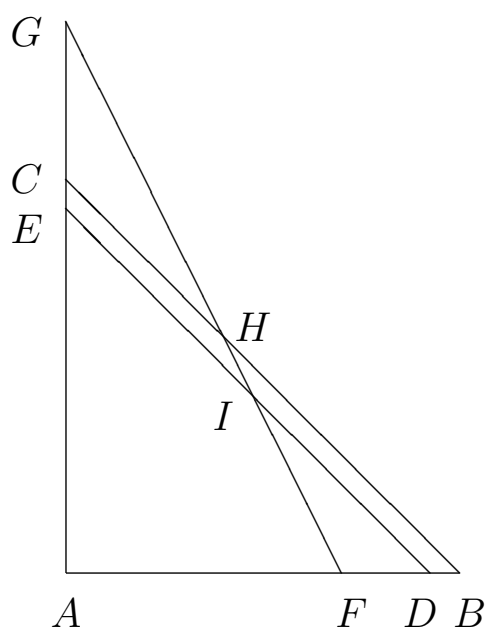

Figure 1. Graphic visualization of Example 1.

Therefore, the IVIF-degree of coincidence of the second pattern with the original pattern will be

$$
\left\langle\left[\frac{a}{s}, \frac{a+e}{s}\right],\left[\frac{b}{s}, \frac{b+c}{s}\right]\right\rangle .
$$

This simple example shows the IVIF-possibility to evaluate more details than the FS- or IFStools, because in the fuzzy set case, the evaluation would to be only $\frac{b}{a}$ and in the IFS-case, it would to be $\left\langle\frac{a}{s}, \frac{b+c}{s}\right\rangle$. 
The degree of uncertainty is determined as the interval

$$
\left\langle\left[0, \frac{d}{s}\right]\right\rangle \text {. }
$$

More complex is the following example.

Example 2. Let us have (see Fig. 2) the original pattern - in the new example, again triangle $A B C$ that must be compared to the other pattern - now triangle $A G I$. Let the sections $B C$ and $G I$ be fuzzified, i.e., they are modified to the regions $B C E D$ and $F G I H$. Let

$$
\begin{gathered}
s=\# A B L I, \\
a=\# A F K E, \\
b=\# F G M K, \\
c=\# G D M, \\
d=\# D B L M, \\
e=\# E K J C, \\
f=\# C J H, \\
g=\# I H J L, \\
h=\# J K M L .
\end{gathered}
$$

Obviously, $a+b+c+d+e+f+g+h=s$.

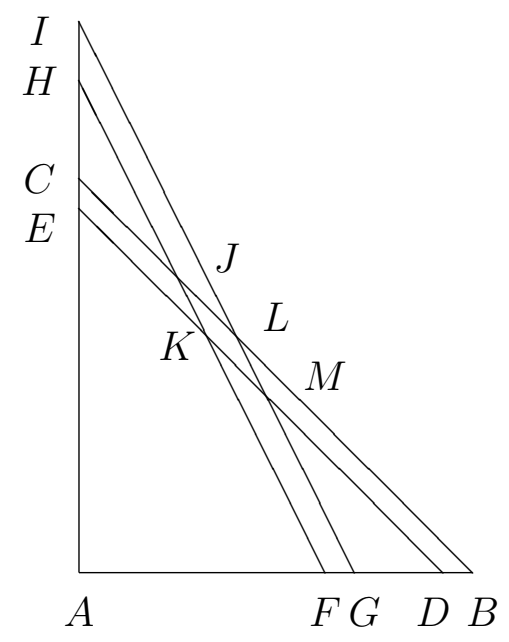

Figure 2. Graphic visualization of Example 2.

Now, the IVIF-degree of coincidence of the second pattern with the original pattern will be

$$
\left\langle\left[\frac{a}{s}, \frac{a+b+e+h}{s}\right],\left[\frac{c}{s}, \frac{c+d}{s}\right]\right\rangle \text {. }
$$

This simple example shows the IVIF-possibility to estimate more detail than the fuzzy set or IFStools, because in the fuzzy set case, the estimation would to be only $\frac{b}{a}$ and in the IFS-case, it would to be $\left\langle\frac{a}{s}, \frac{b+c}{s}\right\rangle$. The degree of uncertainty is determined as the interval $\left\langle\left[0, \frac{f+g}{s}\right]\right\rangle$. 


\subsection{IF-estimations in neural networks and evolutionary algorithms}

The first results related to the IF-estimations in neural networks date back to the year 1990 [32] and they are continued in $[15,16,54-61,64,65]$. These estimations are related, from one side, to the initial values in the input vectors that now will have the form $\left\langle M_{i}, N_{i}\right\rangle$ for the $i$-th input neuron, where $M_{i}, N_{i} \subseteq[0,1]$ and $\sup M_{i}+\sup N_{i} \leq 1$. From the other hand, the weight coefficients of the connections between the nodes with the form $\left\langle V_{i, j}, W_{i, j}\right\rangle$ for the $i$-th and $j$ th neurons lying in sequential layers, where $V_{i, j}, W_{i, j} \subseteq[0,1]$ and $\sup V_{i, j}+\sup W_{i, j} \leq 1$. When some of these coefficients have IF-truth-value $\langle[0,0],[1,1]\rangle$, then we can interpret that the respective object (nodes or arcs between nodes) does not exists. So, we can modify the neural network structure in current time.

In [28], it is mentioned that "The paradigm of Evolutionary Algorithms (EAs) consists of stochastic search algorithms inspired by the process of neo-Darwinian evolution. ... There are several kinds of EAs, such as Genetic Algorithms, Genetic Programming, Classifier Systems, Evolution Strategies, Evolutionary Programming, Estimation of Distribution Algorithms, etc."

In this direction of research, in near future the focus will be oriented to the mentioned above EAs.

In [8], some other areas of DM that can use IF-estimations, were described. All they can use IVIF-estimations, too. Some of these areas are the following.

- machine and e-learning

- clusterisation and classification of data

- knowledge discovery processes

- processes for imputation (filling in) of missing data

and others.

\section{Conclusion}

The present paper aims to offer a new look on different aspects and procedures of DM from the point of view of IVIFSs.

Having in mind that elements of each IVIFSs have four parameters $(\inf M, \sup M, \inf N$, $\sup N$ ), we can mention that in all the commented areas of DM, we see the application of intervalvalued intuitionistic fuzziness as a tool for more precise estimation, which takes into account possibly simultaneously opposite patterns of behaviour, as well as uncertainty.

In [68], an idea for a new direction in AI was formulated by L. Zadeh, based on the concept of a granule. But, by the moment there is not a good formal definition of this concept. Probably, the estimations of the IVIFS-elements can be used for a model. Really, the geometrical interpretation of an IVIFS-element $x$ is given in Figure 3. 


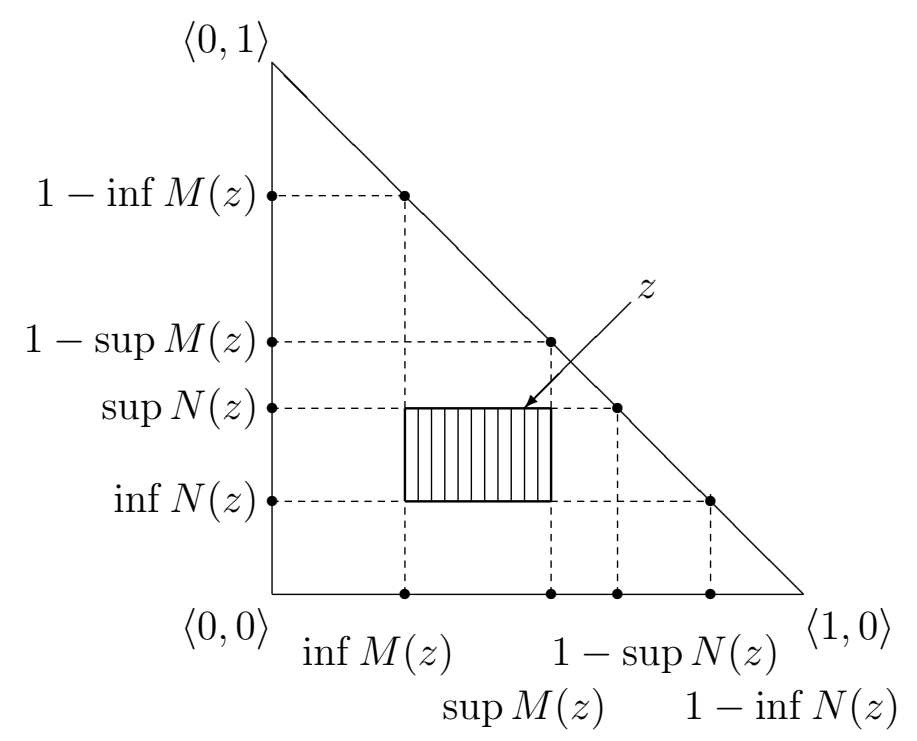

Figure 3. Geometrical interpretation of an IVIFS-element $x$

Let $E$ be a universe, $A=\{\langle x, M(x), M(x)| x \in E\}$ be an IVIFS, and $z \in E$ be a fixed element of the IVIFS. A granule can be defined as the set

$$
G_{\text {crisp }}(z)=\{y \mid y \in E \& \mu(y) \in M(z) \& \nu(y) \in N(z)\}
$$

(crisp form) or

$$
G_{I F S}(z)=\{\langle y, \mu(y), \nu(y)\rangle \mid y \in E \& \mu(y) \in M(z) \& \nu(y) \in N(z)\}
$$

(intuitionistic fuzzy form).

Of course, this is only the first step of the development of this idea that has the potential to develop in future.

\section{Acknowledgements}

The author is thankful for the support provided by the National Scientific Fund of Bulgaria under the Grant DN02/10 "New Instruments for Knowledge Discovery from Data, and their Modelling" and by the Grant BG16RFOP002-1.005-0001 "Increasing the innovation activity of jBoxers LLC. by developing a new innovative product for retrieving and processing information from natural language text" of the Operational Program "Innovation and Competitiveness", 2014-2020.

\section{References}

[1] Angelov, P., Filev, D., \& Kasabov, N. (2010). Evolving Intelligent Systems, John Wiley \& Sons, Hoboken.

[2] Atanassov, K. (1987). Generalized index matrices. Compt. Rend. de l'Academie Bulgare des Sciences, 40 (11), 15-18. 
[3] Atanassov, K. (1988). Review and new results on intuitonistic fuzzy sets. Preprint IMMFAIS-1-88, Sofia, 1988. Reprinted: Int. J. Bioautomation, 20 (S1), S7-S16.

[4] Atanassov, K. (1994). Remark on intuitionistic fuzzy expert systems, BUSEFAL, 59, 71-76.

[5] Atanassov, K. (1998). Generalized Nets in Artificial Intelligence. Vol. 1: Generalized Nets and Expert Systems, "Prof. M. Drinov" Academic Publishing House, Sofia.

[6] Atanassov, K. (1999). Intuitionistic Fuzzy Sets: Theory and Applications, Springer, Heldelberg.

[7] Atanassov, K. (2014). Index Matrices: Towards an Augmented Matrix Calculus, Springer, Cham.

[8] Atanassov, K. (2015). Intuitionistic fuzzy logics as tools for evaluation of Data Mining processes, Knowledge-Based Systems, 80, 122-130.

[9] Atanassov, K. (2018). Intercriteria Analysis over Patterns. Learning Systems: From Theory to Practice (V. Sgurev, V. Piuri, V. Jotsov, Eds.), Studies in Computational Intelligence, Springer, Cham, 61-71.

[10] Atanassov, K. (2018). On the Most Extended Modal Operator of First Type over IntervalValued Intuitionistic Fuzzy Sets. Mathematics, 6, 123; doi:10.3390/math6070123.

[11] Atanassov, K. (2018). Intuitionistic fuzzy sets and interval-valued intuitionistic fuzzy sets. Advanced Studies in Contemporary Mathematics, 28 (2), 167-176.

[12] Atanassov, K., Atanassova, V., \& Gluhchev, G. (2015). InterCriteria Analysis: Ideas and problems. Notes on Intuitionistic Fuzzy Sets, 21 (1), 81-88.

[13] Atanassov, K., \& Gargov, G. (1989). Interval valued intuitionistic fuzzy sets, Fuzzy Sets and Systems, 31 (3), 343-349.

[14] Atanassov, K., Mavrov, D., \& Atanassova, V. (2014). Intercriteria Decision Making: A New Approach for Multicriteria Decision Making, Based on Index Matrices and Intuitionistic Fuzzy Sets. Issues in Intuitionistic Fuzzy Sets and Generalized Nets, 11, 1-8.

[15] Atanassov, K., Sotirov, S., \& Kodogiannis, V. (2006). Intuitionistic fuzzy estimations of the Wi-Fi connections, First Int. Workshop on Intuitionistic Fuzzy Sets, Generalized Nets, and Knowledge Engineering, London, 6-7 Sept. 2006, 75-80.

[16] Atanassov, K., Sotirov, S., \& Krawczak, M. (2009). Generalized net model of the intuitionistic fuzzy feed forward neural network. Notes on Intuitionistic Fuzzy Sets, 15 (2), 18-23.

[17] Atanassov, K., Szmidt, E., Kacprzyk, J., \& Atanassova, V. (2017). An approach to a constructive simplification of multiagent multicriteria decision making problems via intercriteria analysis. Comptes rendus de lAcademie bulgare des Sciences, 70 (8), 1147-1156. 
[18] Berti-Equille, L. (2007). Measuring and modelling data quality for quality-awareness in data mining. In:- Quality Measures in Data Mining (F. Guillet and H. Hamilton, Eds.), Springer, Berlin, 101-126.

[19] Bramer, M. (2013). Principles of Data Mining, Springer, London.

[20] Bull, L., Ester, B.-M., \& Holmes, J. (2008). Learning classifier systems in data mining: An introduction, In:- Learning Classifier Systems in Data Mining (L. Bull, B.-M. Ester, J. Holmes, Eds.), Springer, Berlin, 1-15.

[21] Büyüközkan, G., \& Feyaioğlu, O. (2005). Accelerating the new product introduction with intelligent data mining, In:- Intelligent Data Mining: Techniques and Applications (D. Ruan, G. Chen, E. Kerre, G. Wets, Eds.), Springer, Berlin, 337-354.

[22] Chountas, P., Kolev, B., Rogova, E., Tasseva, V., \& Atanassov, K. (2007). Generalized Nets in Artificial Intelligence. Vol. 4: Generalized Nets, Uncertain Data and Knowledge Engineering. "Prof. M. Drinov" Academic Publishing House, Sofia.

[23] Chountas, P., Sotirova, E., Kolev, B., \& Atanassov, K. (2006). On intuitionistic fuzzy expert systems with temporal components. In:- Computational Intelligence, Theory and Applications. Springer, Berlin, 241-249.

[24] Cios, K., Pedrycz, W., \& Swiniarski, R. (1998). Data Mining Methods for Knowledge Discovery, Kluwer.

[25] Cios, K., Pedrycz, W., Swiniarski, R., \& Kurgan, L. (2007). Data Mining. A Knowledge Discovery Approach, Springer, New York.

[26] Cox, E. (2005). Fuzzy Modeling and Genetic Algorithms for Data Mining and Exploration, Elsevier, Amsterdam.

[27] Dahan, H., Cohen, S., Rokach, L., \& Maimon, O. (2014). Proactive Data Mining with Decision Trees, Springer, New York.

[28] Freitas, A. A. (2010). A Review of Evolutionary Algorithms for Data Mining. In:- Data Mining and Knowledge Discovery Handbook (O. Maimon and L. Rokach, Eds.), 2nd Edition, Springer, New York, 371-400.

[29] Granichin, O., Volkovich, Z., \& Toledano-Kitai, D. (2015). Randomized Algorithms in Automatic Control and Data Mining, Springer, Berlin.

[30] Grosan, V., \& Abraham, A. (2011). Intelligent Systems - A Modern Approach, Springer, Berlin.

[31] Grzymala-Busse, J. W. (2010). Rule induction, In:- Data Mining and Knowledge Discovery Handbook (O. Maimon and L. Rokach, Eds.), 2nd Edition, Springer, New York, 249-265. 
[32] Hadjyisky, L., \& Atanassov, K. (1993). Intuitionistic fuzzy model of a neural network. BUSEFAL, 54, 36-39.

[33] Han, J., \& Kamber, M. (2006). Data Mining: Concepts and Techniques, Morgan Kaufmann.

[34] Hand, D., Mannila, H., \& Smyth, P. (2001). Principles of Data Mining, MIT Press.

[35] Hastie, T., Tibshirani, R., \& Friedman, J. (2001). The Elements of Statistical Learning Data Mining, Inference and Prediction. Springer, New York.

[36] Hilderman, R., \& Peckham, T. (2007). Statistical methodologies from mining potentially interesting contrast sets. In:- Quality Measures in Data Mining (F. Guillet and H. Hamilton, Eds.), Springer, Berlin, 153-177.

[37] Holmes, D., Tweedale, J., \& Jain, L. (2012). Data mining techniques in clustering, association and classification. In:- Data Mining: Foundations and Intelligent Paradigms, Vol. 1: Clustering, Association and Classification (D. Holmes and L. jain, Eds.), Springer, Berlin, $1-6$.

[38] Hong, T.-P., Chen, C.-H., Wu, Y.-L., \& Tseng, V. S. (2008). Fining active membership functions in fuzzy data mining. In:- Data Mining: Foundations and Practice (T.Y. Lin, Y. Xie, A. Wasilewska, C.-J. Liau, Eds.), Springer, Berlin, 179-196.

[39] Kasabov, N. (2007). Evolving Connectionist Systems, Springer, London.

[40] Kecman, V. (2001). Learning and Soft Computing, MIT Press.

[41] Klosgen, W., Zytkow, J. (Eds.) (2002). Handbook of Data Mining and Knowledge Discovery, Oxford University Press, New York.

[42] Kolev, B., El-Darzi, E., Sotirova, E., Petronias, I., Atanassov, K., Chountas, P., \& Kodogiannis, V. (2006). Generalized Nets in Artificial Intelligence. Vol. 3: Generalized Nets, Relational Data Bases and Expert Systems. "Prof. M. Drinov" Academic Publishing House, Sofia.

[43] Maimon, O., \& Rokach, L. (2010). Introduction to knowledge discovery and data mining. In:- Data Mining and Knowledge Discovery Handbook (O. Maimon and L. Rokach, Eds.), 2nd Edition, Springer, New York, 1-15.

[44] Meyer-Nieberg, S., \& Beyer, H.-G. (2007). Self-adaptation in evolutionary algorithms. In:Parameter Setting in Evolutionary Algorithms (F. Lobo, C. Lima, Z. Michalewicz, Eds.), Studies in Computational Intelligence, No. 54, Springer, Berlin, 47-75.

[45] Moyle, S. (2010). Collaborative Data Mining, In:- Data Mining and Knowledge Discovery Handbook (O. Maimon and L. Rokach, Eds.), 2nd Edition, Springer, New York, 1029-1039. 
[46] Orriols-Puig, A., \& Bernado-Mansilla, E. (2008). Mining imbalanced data with learning classifier systems, In:- Learning Classifier Systems in Data Mining (L. Bull, B.-M. Ester, J. Holmes, Eds.), Springer, Berlin, 123-145.

[47] Pechenizkiy, M., Puuronen, S., \& Tsymbal, A. (2008). Does relevance matter to data mining research?, In:- Data Mining: Foundations and Practice (T.Y.Lin, Y. Xie, A. Wasilewska, C.-J. Liau, Eds.), Springer, Berlin, 251-275.

[48] Pena-Ayala, A. (Ed.) (2014). Educational Data Mining, Springer, Cham.

[49] Rokach, L. (2010). A survey of clustering algorithms, In:- Data Mining and Knowledge Discovery Handbook (O. Maimon and L. Rokach, Eds.), 2nd Edition, Springer, New York, 269-298.

[50] Rud, O. P. (2001). Data Mining Cookbook, John Wiley \& Sons, Danvers.

[51] Seifert, J. (2004). Data Mining: An Overview, CRS Report for Congress, Order Code RL31798, Dec. 2004.

[52] Shmueli, G., Patel, N., \& Bruce, P. (2007). Data Mining for Business Intelligence, John Wiley \& Sons, Hoboken.

[53] Simovici, D., \& Djeraba, C. (2014). Mathematical Tools for Data Mining, 2nd Edition, Springer, London.

[54] Sotirov, S. (2007). Determining of intuitionistic fuzzy sets in estimating probability of spam in the e-mail by the help of the neural network, Issues in Intuitionistic Fuzzy Sets and Generalized Nets, 4, 43-47.

[55] Sotirov, S. (2007). Intuitionistic fuzzy estimations for connections of the transmit routines of the bluetooth interface, Advanced Studies in Contemporary Mathematics, 15 (1), 99-108.

[56] Sotirov, S. (2007). Method for determining of intuitionistic fuzzy sets in discovering water floods by neural networks, Issues in Intuitionistic Fuzzy Sets and Generalized Nets, 4, 9-14.

[57] Sotirov, S., \& Atanassov, K. (2009). Intuitionistic fuzzy feed forward neural network. $C y$ bernetics and Information Technologies, 9 (2), 62-68.

[58] Sotirov, S., \& Atanassov, K. (2012). Generalized Nets in Artificial Intelligence. Vol. 6: Generalized Nets and Supervised Neural Networks. "Prof. M. Drinov" Academic Publishing House, Sofia.

[59] Sotirov, S., \& Dimitrov, A. (2010). Neural network for defining intuitionistic fuzzy estimation in petroleum recognition, Issues in Intuitionistic Fuzzy Sets and Generalized Nets, 8, 74-78. 
[60] Sotirov, S., Kodogiannis, V., \& Elijah Blessing, R. (2006). Intuitionistic fuzzy estimations for connections with Low Rate Wireless personal area networks, First Int. Workshop on on Intuitionistic Fuzzy Sets, Generalized Nets, and Knowledge Engineering, London, 6-7 Sept. 2006, 81-87.

[61] Sotirov, S., Vardeva, I., \& Krawczak, M. (2013). Intuitionistic fuzzy multilayer perceptron as a part of integrated systems for early forest-fire detection, Notes on Intuitionistic Fuzzy Sets, 19 (3), 81-89.

[62] Spurgin, A., \& Petkov, G. (2005). Advances simulator data mining for operators' performance assessment, In:- Intelligent Data Mining: Techniques and Applications (D. Ruan, G. Chen, E. Kerre, G. Wets, Eds.), Springer, Berlin, 487-514.

[63] Sumathi, S., \& Sivanandam, S. (2006). Introduction to Data Mining and Applications, Berlin.

[64] Valchev, D., Sotirov, S. (2009). Intuitionistic fuzzy detection of signal availability in multipath wireless chanels, Notes on Intuitionistic Fuzzy Sets, 15 (2), 24-29.

[65] Vardeva, I., Sotirov, S. (2009). Intuitionistic fuzzy estimation of damaged packets with multilayer perceptron, Proc. of the Tenth International Workshop on Generalized Nets, IWGN'2009, Sofia, 63-69.

[66] Witten, H., \& Frank, E. (2005). Data Mining: Practical Machine Learning Tools and Techniques, Morgan Kaufmann.

[67] Yao, Y., Zhong, N., Zhao, Y. (2008). A conceptual framework of data mining, In:- Data Mining: Foundations and Practice (T. Y. Lin, Y. Xie, A. Wasilewska, C.-J. Liau, Eds.), Springer, Berlin, 501-515.

[68] Zadeh, L. (1965). Fuzzy sets. Information and Control, 8, 338-353. 